Copyright 2007 Society of Photo-Optical Instrumentation Engineers.

This paper published in Journal of Electronic Imaging and is made available as an electronic reprint with permission of SPIE. One print or electronic copy may be made for personal use only. Systematic or multiple reproduction, distribution to multiple locations via electronic or other means, duplication of any material in this paper for a fee or for commercial purposes, or modification of the content of the paper are prohibited.

\title{
Quantitative Perceptual Comparison of Variable Bit Rate over Constant Bit Rate Encoding Scheme for MPEG-4 Video
}

\author{
H. Koumaras ${ }^{1}$, G. Gardikis ${ }^{2}$, A. Kourtis ${ }^{2}$, D. Martakos ${ }^{1}$ \\ ${ }^{1}$ University of Athens, Informatics and Telecommunications Department \\ Panepistimioupoli, Zografou, Attiki, Athens, Greece \\ $\{$ koumaras, martakos\}@di.uoa.gr \\ ${ }^{2}$ Institute of Informatics and Telecommunications NCSR "DEMOKRITOS" \\ Patriarchou Gregoriou Str., Agia Paraskevi, Attiki, 15310 Athens Greece \\ \{koumaras, gardikis, kourtis\}@ iit.demokritos.gr
}

\begin{abstract}
Between the two main schemes of digital video coding, VBR encoding scheme is generally considered as better in terms of efficiency and encoding quality in comparison to $\mathrm{CBR}$, because it retains the same quantization parameters for the whole encoding procedure (unconstrained VBR), without altering them according to a specific adaptive rate algorithm. Towards this generally accepted statement, this paper presents a quantitative comparison to the perceptual efficiency of VBR over CBR for Moving Picture Expert Group - 4 (MPEG-4) ASP CIF and QCIF encoding sequences, showing that the VBR does not out-perform significantly the corresponding CBR encoding quality, since the deduced perceptual advantage/ratio of VBR over CBR for CIF is approximately $4-5 \%$ and constant for all the encoding bit rates greater than $200 \mathrm{kbps}$, while for the QCIF-case the relative ratio drops to approximately $2.5 \%$.

Keywords: Video Quality, CBR, VBR, MPEG-4
\end{abstract}




\section{Introduction}

From the advent of video coding, two main encoding schemes were proposed and are still in use: The Constant Bit Rate (CBR) and the Variable Bit Rate (VBR). The VBR mode retains the same quantization parameters for all the encoding process in contrast to the CBR mode, which adapts the quantization parameters dynamically according to a sophisticated rate algorithm in proportion to the spatial and temporal activity of the encoding video signal.

Today, the choice of VBR mode for video services over communication networks generally prevails over CBR mode due to a number of advantages such as [1]:

ß Better video quality for the same average bit rate without the complexity of an adaptive rate algorithm

BShorter transmission delay since the buffer size in the encoder side can be reduced without encountering an equivalent delay in the network

B Increased call-carrying capacity due to the fact that the bandwidth per call for VBR video may be lower than for equivalent quality of CBR source.

ßBetter exploitation of the available capacity of the transmission channel by the statistical multiplexing of VBR streams in comparison to CBR streams.

Thus, it is generally supported that more efficient network utilization can be achieved by choosing VBR encoding mode.

However, except for the pure network-based selection of the encoding mode (i.e. CBR or VBR), the fact that perceived quality degradation is caused by the digital video compression process, it has raised the issue of the user satisfaction (i.e. perceived video quality level or perceived quality of service) [2] in correlation with the selected encoding parameters.

Among the various encoding parameters that play significant role in the deduced perceived quality of service (PQoS) (e.g. bit rate, spatial and temporal resolution), the selection of VBR or CBR encoding mode is critical for the final perceptual outcome. Although a lot of research has been focused on developing techniques and methods for estimating the video quality of a compressed/encoded video signal [3-16], the issue of studying quantitatively the perceptual efficiency of VBR over CBR mode has not been performed yet. The engineers generally select VBR over CBR due to the aforementioned theoretical advantages of the first, usually using simply the PSNR metric for measuring its encoding efficiency, without taking under consideration the actual perceptual impact of VBR over CBR.

This paper presents a quantitative study on the perceptual effectiveness of VBR over CBR in correlation to the encoding bit rate, considering that the rest encoding parameters (e.g. spatial and temporal resolution, encoding scheme, GOP pattern etc.) remain constant. Towards this, we provide results, depicting the actual perceived efficiency of VBR/CBR modes and not only the engineering effectiveness of each one, which may be measurable by simple error-based metrics but not actually perceived by the human visual system.

The rest of the paper is organized as follows: Section 2 discusses the various video quality metrics and methods that have been proposed in the literature and explains the research method, which this paper has followed. Section 3 presents the experimental results of VBR/CBR video quality evaluation, quantifying the perceptual efficiency of 
VBR over CBR for both CIF and QCIF resolution. Finally, section 4 concludes the paper, discussing the outcomes of this work.

\section{Video Quality Metrics}

The evaluation of the video quality is a matter of objective and subjective procedures, which take place after the encoding process (post-encoding evaluation). Subjective quality evaluation processes of video streams require large amount of human resources, establishing it as a time-consuming process (e.g. large audiences evaluating video/audio sequences) [3],[4],[5]. Objective evaluation methods, on the other hand, can provide PQoS evaluation results faster, but require large amount of machine resources and sophisticated apparatus configurations.

The majority of the existing objective methods in the literature requires the undistorted source video sequence as a reference entity in the quality evaluation process, and due to this, these methods are characterized as Full Reference (FR) [6-13]. Basically, these methods are based on an error sensitivity framework between the uncompressed and the encoded video signal, while frame-by-frame comparison is performed. When the original undistorted/uncompressed video signal is not required for the video quality evaluation process, then the objective method is characterized as Non-Reference (NR) [14-15]. Recently, some works have been published, which exploit some already proposed FR or NR metrics in order to predict the final perceived quality of a specific encoded sequence at a pre-encoding stage [16-18].

For the needs of this paper, in order to quantify the perceptual difference between the CBR and VBR encoding efficiency, the use of objective instead of subjective procedures was preferred. Since the perceptual difference between the two encoding schemes is expected to be rather small, the subjective assessments could not be able to provide a reliable result due to the relative high statistical error [22], which will encompass the corresponding evaluation. Keeping this restriction in mind, we decided to use four objective FR metrics in the evaluation procedure: the FR Peak Signal-to-Noise Ratio (PSNR), the FR Structural SIMilarity (SSIM) [12],[13] the FR Digital Video Quality (DVQ) [8] and the FR Delta metric [19]. These four efficient FR objective metrics will provide accurate and specific results for the perceptual efficiency of the encoding schemes, without introducing significant statistical errors, which may cause error propagation in the rest processing, eliminating therefore the accuracy of our results. Information about the correlation of these objective metrics to subjective evaluation procedures can be found at [21] (for more detailed description, please see the relative references). For completeness of the paper, we briefly describe hereby each metric:

\section{- $\quad$ PSNR}

Peak Signal to Noise ratio is one of the more tangible parameters used to measure video quality. The specific metric is provided during the compression process by the encoder and is defined as follows:

$$
P S N R=10 \log _{10} \frac{L^{2}}{\mathrm{MSE}}
$$


where $\mathrm{L}$ denotes the dynamic pixel value (i.e. equal to 255 for 8 bits/pixel monotonic signal) and the Mean Square Error is defined as

$$
M S E=\frac{1}{N} \sum_{i=1}^{N}\left(x_{i}-y_{i}\right)^{2}
$$

where $N$ denotes the number of pixels of the selected spatial resolution and $x_{i} / y_{i}$ the $i^{\text {th }}$ pixel value in the original/encoded frame.

- SSIM

SSIM is a novel FR metric for measuring the structural similarity between two image sequences, exploiting the general principle that the main function of the human visual system is the extraction of structural information from the viewing field. If $x$ and $y$ are two video frames, then the SSIM is defined as:

$$
\operatorname{SSIM}(x, y)=\frac{\left(2 \mu_{x} \mu_{y}+C_{1}\right)\left(2 \sigma_{x y}+C_{2}\right)}{\left(\mu_{x}^{2}+\mu_{y}^{2}+C_{1}\right)\left(\sigma_{x}^{2}+\sigma_{y}^{2}+C_{2}\right)}
$$

where $\mu_{x}, \mu_{y}$ are the mean of $x$ and $y, \sigma_{x}, \sigma_{y}, \sigma_{x y}$ are the variances of $x, y$ and the covariance of $x$ and $y$, respectively. The constants $C_{1}$ and $C_{2}$ are defined as:

$$
C_{1}=\left(K_{1} L\right)^{2} C_{2}=\left(K_{2} L\right)^{2}
$$

where $L$ is the dynamic pixel range and $K_{1}=0.01$ and $K_{2}=0.03$, respectively [12-13].

\section{- Delta}

It is a grey-scale measure, which calculates the distances in either discrete or continuous space. The distance between two picture functions can be defined as the mathematical distance between the volumes beneath the two functions. Considering that $f$ and $g$ are two images defined in the pixel raster X, featuring the same grey levels, with $\Gamma_{f}$ and $\Gamma_{g}$ being the subgraphs of $f$ and $g$ respectively [19], then the Delta metric $\Delta \mathrm{g}$ is defined as

$$
\Delta_{g}\left(\Gamma_{f}, \Gamma_{g}\right)=\left[\frac{1}{n(X) n(Y)} \sum_{x \in X} \sum_{y \in Y}\left|d\left((x, y), \Gamma_{f}\right)-d\left((x, y), \Gamma_{g}\right)\right|^{p}\right]^{1 / p} \text { for } 1 \leq p \leq \infty
$$

\section{- DVQ}

This metric is based on the Discrete Cosine Transform. It incorporates aspects of early visual processing, including light adaptation, luminance and chromatic channels, spatiotemporal filtering, spatial frequency channels, contrast masking, and probability summation [8]. DVQ uses the Discrete Cosine Transform (DCT) in order to perform decomposition of the original data into spatial channels. This provides a powerful advantage towards the implementation of this metric, since efficient hardware and software are available for this transformation and because in many applications the transform may have already been done as part of the compression process.

The use of these four FR objective quality metrics for the video quality evaluation process ensures the accuracy of the deduced results, because possible limitations of each 
metric on the deduced measurements are generally eliminated by the use of the rest metrics.

\section{Video Quality Evaluation}

For the experiments of this paper the ISO MPEG-4 codec implementation developed by Dicas Corporation was used. Dicas Corporation is an official member of the MPEG Industry Forum and a supporter of the AVC Alliance, denoting close relation of this implementation to the standard. Regarding the selection of the profile (the profile in MPEG-4 is a set of tools that define a conformance point), although the presented experimental results could be derived for any MPEG-4 visual profile (Simple, Core, Main, Advanced Simple Profile), the Advanced Simple Profile was selected, because it is the most widely used profile in MPEG-4 commercial applications (e.g. mobile phones, cameras, codec software/hardware etc.), while the rest profiles are mainly restricted to the MPEG-4 reference encoder implementation. Moreover, the Simple, Core and Main Profiles are subsets of the Advanced Simple Profile, which means that any video stream that complies to the Simple, Core and Main Profiles, also complies with the Advanced Simple Profile. More specifically, the ASP specifications fit well with the selected spatial resolution values (i.e. CIF and QCIF), supporting all the main features of MPEG4, such as the Basic Visual Tools, Error resilience, short header, B-VOP, Method 1/2 quantization and $1 / 2$ Pel pixel motion compensation. Thus, the selection of ASP for our experiments is in many ways beneficial and interesting.

For the evaluation process of the video quality, five reference video clips were used (i.e. Suzie, Cactus, Flower Garden, Table-Tennis, Mobile \& Calendar), which represent a wide range of spatial and temporal activity level. These video clips were encoded from their original uncompressed format to ISO MPEG-4 CIF resolution Advanced Simple Profile at various bit rates (i.e. 100, 150, 200, 250, 300, 400, $500 \mathrm{kbps}$ ), using both CBR and VBR encoding modes.

At the encoder, the encoding parameters were selected as follows: The MPEG GOV Structure was set with Key Frame Period 100 frames and B frame period 2 frames. The trade off between compression quality efficiency and the encoding speed was set at "High Quality". Regarding the CBR mode, a VBV Buffer implementation was used with buffer size equal to 1 second.

From each test sequence, 20 randomly selected decoded frames were extracted, which were used for the video quality evaluation/comparison between the VBR and CBR mode for all the encoding bit rates (i.e. 100-500 kbps). The selected decoded frames belonged to either I, P or B frame type, ensuring by this way the testing of both Intra and Intercoding efficiency.

In order to provide a normalization of the video quality evaluation scale of each metric, for the five video test signals the ratio of the perceived quality level of VBR (PQVBR) over the perceived quality level of CBR (PQCBR) is calculated for all the testing encoding bit rates. Thus, for each quality metric, the PQVBR/PQCBR ratio, is derived for the whole test signal set, which is normalized and independent of the quality scale of each metric. Also, for those metrics (i.e. VQM and Delta), which do not follow an ascending scale (i.e. the deduced quality value becomes lower for better encoding quality), the inverse values have been used in the calculation of PQVBR/PQCBR ratio in order to 
provide common results with the rest metrics, where the higher values correspond to better encoding quality and vice versa.

For each $x_{k}$ of the deduced PQVBR/PQCBR ratios at any $m^{\text {th }}$ encoding bit rate for all the $i$ test sequences, it is then derived the corresponding mean value and the standard deviation of each $k$ objective metric, according to the following equations:

$$
\bar{x}_{k}=\frac{1}{N} \sum_{i=1}^{N} x_{i}, \delta x_{k}=\sqrt{\frac{\sum_{i=1}^{N}\left(x_{i}-\bar{x}\right)^{2}}{N(N-1)}} .
$$

Thus, each $k$ objective metric provides a single measurement for PQVBR/PQCBR ratio for all the test sequences at any given $m^{\text {th }}$ bit rate, of the form $x_{k}=\bar{x}_{k} \pm \delta x_{k}$. Moreover, in order to retain the accuracy of the deduced results, the Chauvenet Criterion was also applied on the experimental values, keeping or discarding suspect inaccurate values.

In this experimental framework, Figures 1-4, depict the deduced PQVBR/PQCBR ratio of each quality metric for the whole test signal set, along with the corresponding standard deviations. Between the various experimentally derived points of each figure, the best-fit exponential curve of each case has been drawn in order to display the general tendency of the PQVBR/PQCBR ratio. As it can be derived by all the cases, the perceptual efficiency of the VBR mode over CBR is increasing along with the encoding bit rate. More specifically, the VBR generally seems to outperform the CBR, except for very low encoding bit rates (i.e. $<125 \mathrm{kbps}$ ). Moreover, as it can be seen by the experimentally derived points that the PQVBR/PQCBR ratio tends to remain practically constant for high bit rates.

In order to demonstrate the variance in the perceived quality efficiency between the PQVBR and PQCBR, figure 5 depicts two representative frames taken from the Suzie sequence encoded at ASP CIF/200kbps with CBR -Fig. 5a- and VBR -Fig. 5b- mode respectively (the rest encoding configuration is the aforementioned one). Although at first sight no significant difference is noticeable, a closer observation at the two frames -Fig. $5 \mathrm{c} / \mathrm{d}$ - can reveal a slight perceptual advantage of VBR over CBR.

In order to derive a generic quantitative result of this perceptual efficiency of VBR over CBR for the CIF-case, a single graph is extrapolated by figures 1-4, which combines into one curve the assessments of each individual quality metric curve.

In order to combine the discrete PQVBR/PQCBR ratios of the various metrics into a common estimation, we use from the error theory the following combining method:

Considering the $x_{1}, x_{2}, \ldots, x_{4} \mathrm{PQVBR} / \mathrm{PQCBR}$ ratios of the various video quality metrics at any $m^{\text {th }}$ encoding bit rate, with $x_{k}=\bar{x}_{k} \pm \delta x_{k}$ where $k=\{1,2,3,4\}$, we estimate a generic $X_{m}+d X_{m}$ PQVBR/PQCBR ratio, based on the following equations

$$
X_{m}=\frac{\sum_{k=1}^{N} w_{k} x_{k}}{\sum_{k=1}^{N} w_{k}}, \text { where } w_{k}=1 /\left(\delta x_{k}\right)^{2} \text { and } \delta X_{m}=\sqrt{1 / \sum_{k=1}^{N} w_{k}} \text {. }
$$


By applying this method, the experimental points of PQVBR/PQCBR ratio in figures 1-4, which have been derived using the four NR objective quality metrics, provide the generic curve of figure 6 and the values of Table 1. The curve of fig. 6 depicts the PQVBR perceptual efficiency over PQCBR for the case of MPEG4 ASP/CIF. As it can be observed, the PQVBR/PQCBR ratio for the encoding bit rate area of [100,500] kbps can be successfully approximated by a quadratic order polynomial of the form

$$
y=-0.0071 x^{2}+0.014 x+1,100 \leq x \leq 500 \text { (1) }
$$

where $\mathrm{y}$ is the $\mathrm{PQVBR} / \mathrm{PQCBR}$ ratio and $\mathrm{x}$ is the encoding bit rate.

Thus by using equation 1 , it is possible to be analytically calculated the perceptual efficiency of VBR over CBR for the MPEG-4 ASP/CIF case at a pre-encoding stage. The efficiency of the specific theoretical approximation can be observed by the corresponding residuals, shown in figure 6 , where the norm of the residuals is 0.022414 , demonstrating good match between the experimental and the theoretical results.

Elaborating more on the deduced results of the PQVBR/PQCBR ratio, it must be noted that the PQVBR outperforms the PQCBR almost for the whole encoding bit rate area (except for the very low ones i.e. $<100 \mathrm{kbps}$ ), but the corresponding better perceptual efficiency of PQVBR is not more than $4-5 \%$ in comparison to the PQCBR. More specifically, figure 6 shows that the efficiency of PQVBR over PQCBR remains practically constant for bit rates $>200 \mathrm{kbps}$, proving by this way its better - but limited perceptual efficiency.

For consistency of the paper, we repeated the same experimental procedure, using the same reference video clips, for the case of QCIF VBR/CBR at 32 and 64kbps. The rest encoding parameters were remained constant and similar to the aforementioned CIF case. The used reference and encoded sequences are available online for downloading at [20]. Then, the deduced encoded streams were used as inputs to the four video quality metrics (i.e. PSNR, SSIM, Delta and VQM). Afterwards, the PQVBR/PQCBR ratio was calculated for each case, following exactly the same experimental procedure. Table 2 depicts the deduced results, which show that even for the case of QCIF the perceptual efficiency of VBR over CBR is retained at similar and even lower to CIF-case levels (i.e. around $2.5 \%$ ).

\section{Conclusions}

This paper has presented an analytical and quantitative approach of the perceptual efficiency of VBR encoding mode over CBR for MPEG-4 ASP/CIF-QCIF encoded sequences. It shows that VBR mode provides better perceptual quality than CBR, but at a constant-limited percentage of approximately $4-5 \%$ in comparison to CBR case for CIF resolution and approximately $2.5 \%$ for QCIF case. Thus, it has been verified that the generally stated opinion that VBR provides better encoding quality than CBR, although it is true from an engineering point of view, from the perceptual aspect (i.e. user perception and satisfaction) it is practically limited to only a small perceptual enhancement of VBR over CBR. 


\section{ACKOWLEDGEMENT}

The authors want to acknowledge the contribution of the student Spyridon Papagiakoumos at performing the experimental part of this paper. They would also like to thank the anonymous reviewers for their helpful and constructive comments on the submitted manuscript, which improved the presentation and the quality of this paper. Finally, the work in this paper was carried out within the research framework of FP6027457 IST-IMOSAN project (http://www.ist-imosan.gr)

\section{References}

[1] T. V. Lacshman, A. Ortega, A. R. Reibman, "VBR Video: Tradeoffs and Potentials", Invited Paper, Proceedings of the IEEE, Vol.86, No. 5, May 1998.

[2] F. Liberal, A. Ferro, J. O. Fajardo, "PQoS Based Model for Assessing Significance Statistically" Third International Working Conference on Performance Modelling and Evaluation of Heterogeneous Networks (HET-NETs '05), Ilkley, U.K. July 18-20, 2005.

[3] F. Pereira and T. Alpert, "MPEG-4 Video Subjective Test Procedures and Results", IEEE Transactions on Circuits and Systems for Video Technology. Vol. 7(1), pp. 32-51, 1997.

[4] Th. Alpert and L. Contin, "DSCQE Experiment for the Evaluation of the MPEG-4 VM on Error Robustness Functionality", ISO/IEC - JTC1/SC29/WG11, MPEG 97/M1604, 1997.

[5] ITU "Methology for the subjective assessment of the quality of television pictures", Recommendation ITU-R BT.500-10, 2000.

[6] K. T. Tan and M. Ghanbari, "A Multi-Metric Objective Picture Quality Measurements Model for MPEG Video", IEEE Transactions on Circuits and Systems for Video Technology, Vol.10(7), pp. 1208-1213, 2000.

[7] St. Wolf and M. H. Pinson, "Spatial - Temporal Distortion Metrics for in-service Quality Monitoring of any Digital Video System", SPIE International Symposium on Voice, Video, and Data Communications, Boston, 1999 pp. 11-22

[8] A. B. Watson, J. Hu and J. F. McGowan, "DVQ: A Digital Video Quality Metric Based on Human Vision", Journal of Electronic Imaging, Vol. 10(1), pp. 20-29, 2001.

[9] S. Daly, "The Visible Difference Predictor: An algorithm for the Assessment of Image Fidelity", in Proceedings SPIE, Vol. 1616, pp. 2-15, 1992.

[10] A. P. Bradley, "A Wavelet Difference Predictor", IEEE Transactions on Image Processing. Vol. 5, pp. 717-730, 1999.

[11] Y. K. Lai and J. Kuo, “A Haar Wavelet Approach to Compressed Image Quality Measurement", Journal of Visual Communication and Image Understanding, Vol. 11, pp. 81-84, 2000.

[12] Z. Wang, L. Lu, A. C. Bovik, "Video Quality Assessment Based on Structural Distortion Measurement", Signal Processing: Image Communication, special issue on Objective video quality metrics, Vol. 19(2), pp. 121-132, 2004.

[13] Z. Wang, A. C. Bovik, H. R. Sheikh and E. P. Simoncelli, "Image quality assessment: From error visibility to structural similarity", IEEE Transactions on Image Processing, Vol. 13(4), pp. 1-14, 2004.

[14] J. Lauterjung, "Picture Quality Measurement", Proceedings of the International Broadcasting Convention (IBC), Amsterdam, 1998, pp. 413-417. 
[15] L. Lu, Z. Wang, A. C. Bovik and J. Kouloheris, "Full-reference video quality assessment considering structural distortion and no-reference quality evaluation of MPEG video", IEEE International Conference on Multimedia, 2002.

[16] H. Koumaras, E. Pallis, G. Xilouris, A. Kourtis, D. Martakos, and J. Lauterjung, "Pre-Encoding PQoS Assessment Method for Optimized Resource Utilization," presented at HET-NETs04, Ilkley, West Yorkshire, U.K., 2004.

[17] H. Koumaras, A. Kourtis, D. Martakos, "Evaluation of Video Quality Based on Objectively Estimated Metric", Journal of Communications \& Networking, KICS, Vol.7(3), 235-242, Sep. 2005

[18] H. Koumaras, D. Martakos, A. Kourtis, ““'Objective Measurement of Perceived QoS for Homogeneous MPEG-4 Video Content", Encyclopedia of Multimedia Technology and Networking, pp.770-776, Idea Group Pub. ISBN 1-59140-561-0 (hard cover) -ISBN 1-59140-796-6 (ebook), May 2005

[19] A.J. Baddeley, An error metric for binary images, in: W. Forstner, S. Ruwiedel (Eds.), Proceedings of Robust Computer Vision, 1992, pp. 59 -- 78.

[20] Sequences are available online at http://aias.iit.demokritos.gr/ koumaras/CBR_VBR

[21] VQEG. "Final Report From the Video Quality Experts Group on the Validation of Objective Models of Video Quality Assessment", http://www.vqeg.org. 2000

[22] M. Pinson and S. Wolf, "Comparing subjective video quality testing methodologies" Visual Communications and Image Processing 2003. Edited by Ebrahimi, Touradj; Sikora, Thomas. Proceedings of the SPIE, Volume 5150, pp. 573-582 (2003). 


\begin{tabular}{|c|c|}
\hline Bit Rate (kbps) & Final Weighted Average \\
\hline 100 & $1.01124 \pm 0.00891$ \\
\hline 150 & $1.00307 \pm 0.01146$ \\
\hline 200 & $1.03195 \pm 0.01538$ \\
\hline 250 & $1.01919 \pm 0.01158$ \\
\hline 300 & $1.03337 \pm 0.01159$ \\
\hline 400 & $1.04887 \pm 0.01747$ \\
\hline 500 & $1.03026 \pm 0.00997$ \\
\hline
\end{tabular}

Table 1. The generic CIF PQVBR/PQCBR ratio

\begin{tabular}{|c|c|c|}
\hline $\begin{array}{c}\text { Video Quality } \\
\text { Metric }\end{array}$ & $\begin{array}{c}\text { PQVBR/PQCBR ratio } \\
\text { 32kbps }\end{array}$ & $\begin{array}{c}\text { PQVBR/PQCBR ratio } \\
\text { 64kbps }\end{array}$ \\
\hline PSNR & $1.0218 \pm 0.0069$ & $1.0314 \pm 0.0037$ \\
\hline SSIM & $1.0224 \pm 0.0044$ & $1.0153 \pm 0.0041$ \\
\hline Delta & $1.1026 \pm 0.0573$ & $1.1224 \pm 0.0317$ \\
\hline VQM & $1.0720 \pm 0.0351$ & $1.0832 \pm 0.0201$ \\
\hline Average & $\mathbf{1 . 0 2 3 1 \pm 0 . 0 0 3 7}$ & $\mathbf{1 . 0 2 5 9 \pm 0 . 0 0 2 7}$ \\
\hline
\end{tabular}

Table 2. The PQVBR/PQCBR ratio for the QCIF case 


\section{List of Figures Captions}

Figure 1. The PQVBR/PQCBR ratio of SNR metric for the whole test-signal set

Figure 2. The PQVBR/PQCBR ratio of SSIM metric for the whole test-signal set

Figure 3. The PQVBR/PQCBR ratio of DELTA metric for the whole test-signal set

Figure 4. The PQVBR/PQCBR ratio of DVQ metric for the whole test-signal set

Figure 5. PQVBR/PQCBR comparison between two representative frames from video sequence Suzie

Figure 6. The PQVBR/PQCBR ratio of MPEG4 ASP/CIF and the corresponding residuals, between the experimental and theoretical curve. 


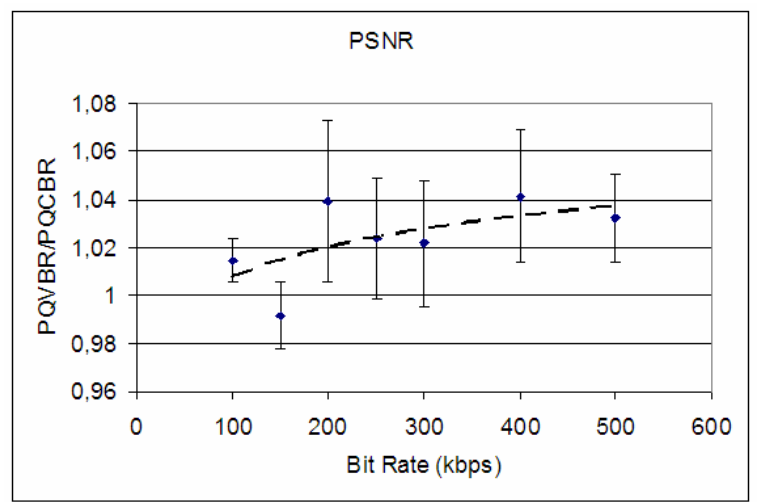

Fig.1

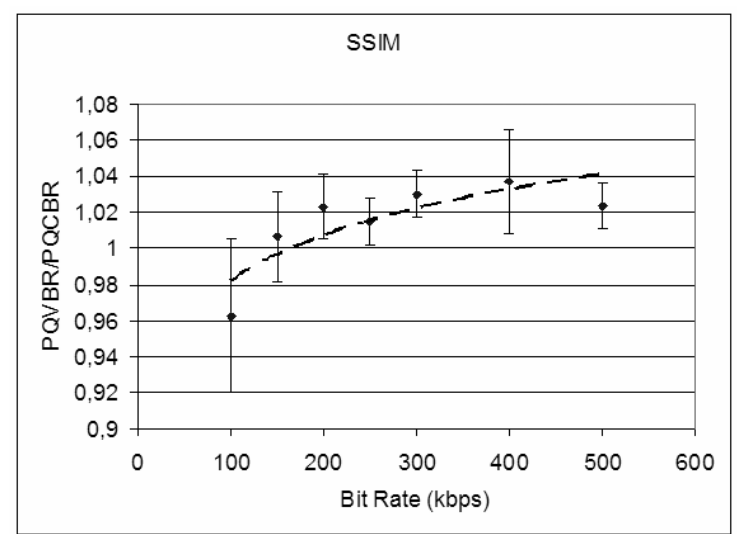

Fig.2

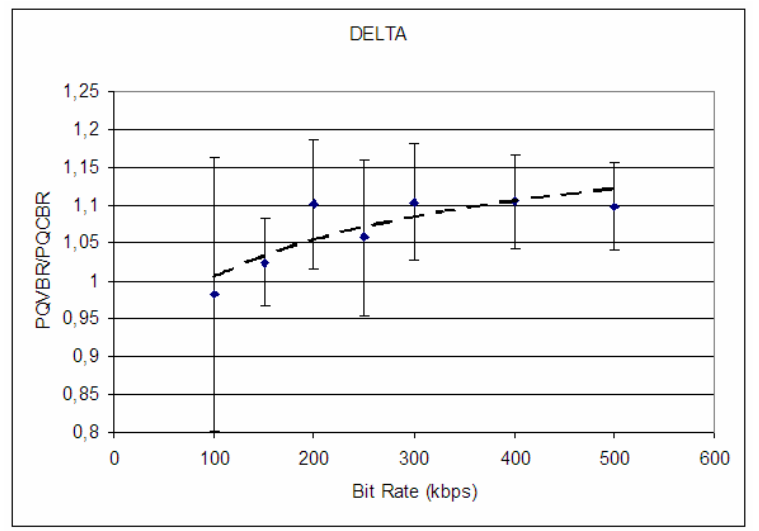

Fig.3 


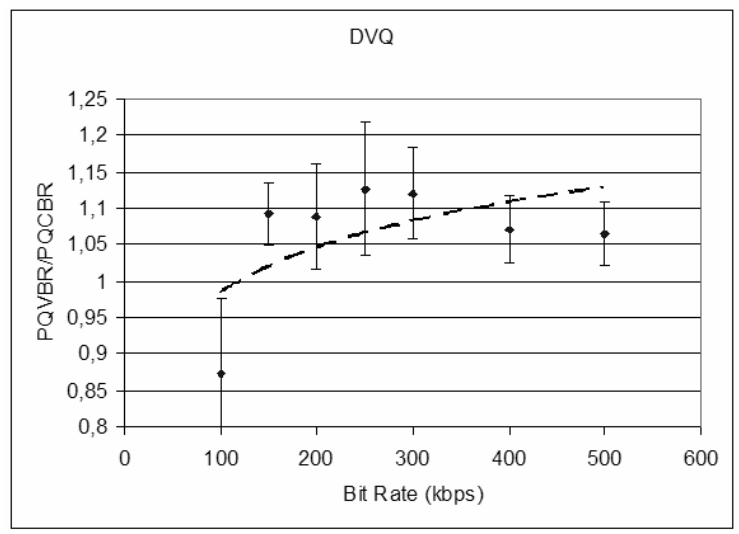

Fig.4
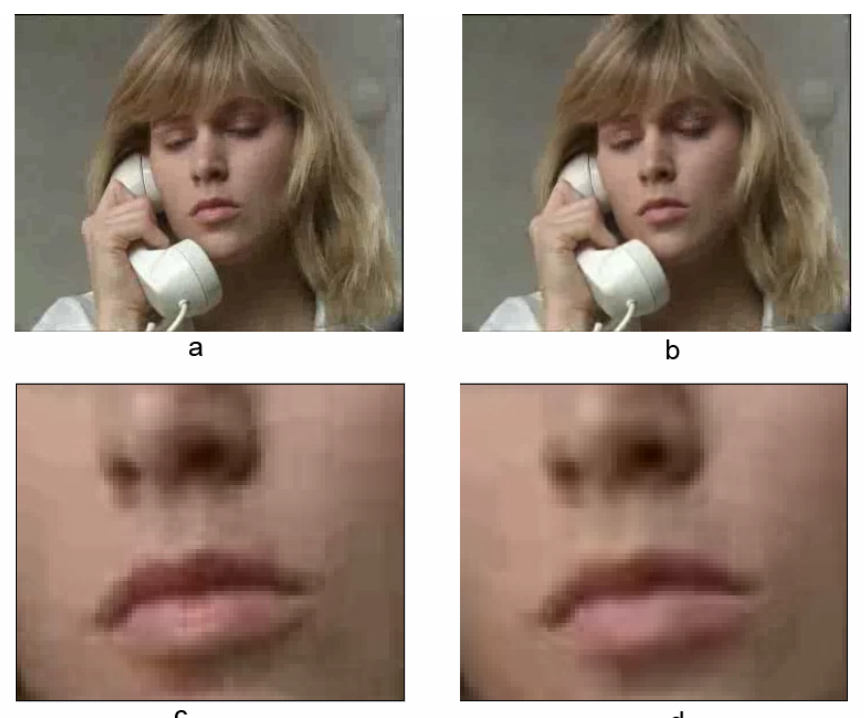

Fig.5 

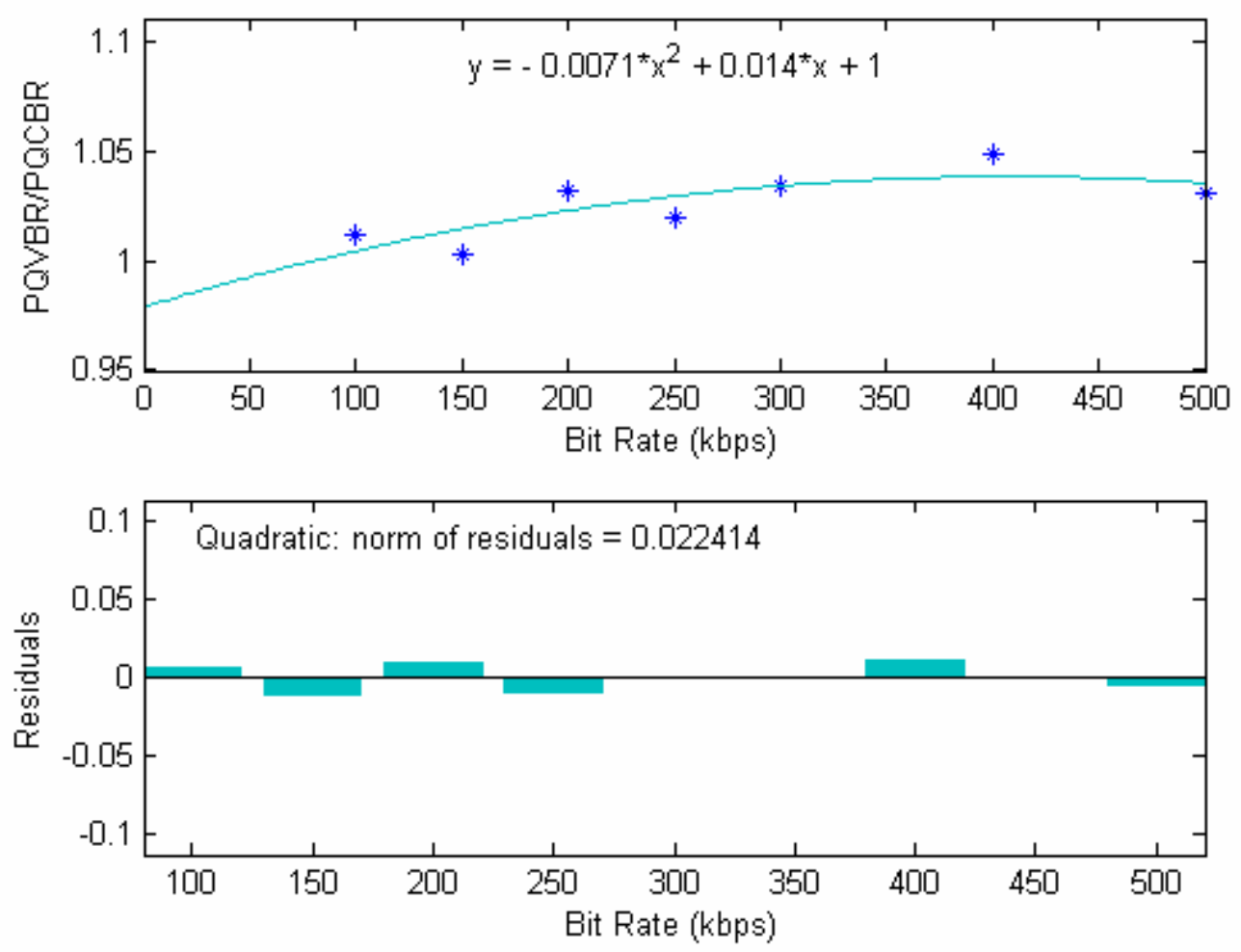

Fig.6 


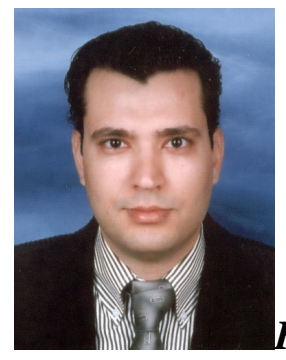

Harilaos Koumaras was born in Athens, Greece in 1980. He received his BSc degree in Physics in 2002 from the University of Athens, Physics Department and his MSc in Electronic Automation and Information Systems in 2004, being scholar of the non-profit organization Alexander S Onassis, from the University of Athens, Physics and Informatics Department. He has also granted with honors the classical piano and harmony degrees from the classical music department of Attiko Conservatory. He joined the Digital Telecommunications Lab at the National Centre of Scientific Research "Demokritos" in 2003 and since then he has participated in the EU funded projects SOQUET, ATHENA, ENTHRONE and national funded project PYTHAGORAS II with presentations and publications at international conferences, scientific journals and book chapters. Currently, he is working toward the $\mathrm{PhD}$ degree, having granted the scholarship of NCSR "Demokritos" for Doctorate dissertation. At the same time, he is an associate lecturer at the Business College of Athens (BCA), teaching modules related to Information Technology. $\mathrm{He}$ also serves as an associate editor the Journal of Telecommunication Systems, published by Springer. His research interests include objective/subjective evaluation of the perceived quality of multimedia services, picture quality evaluation, video traffic modeling, digital terrestrial television and video compression techniques. He is a member of IEEE and National Geographic Society.

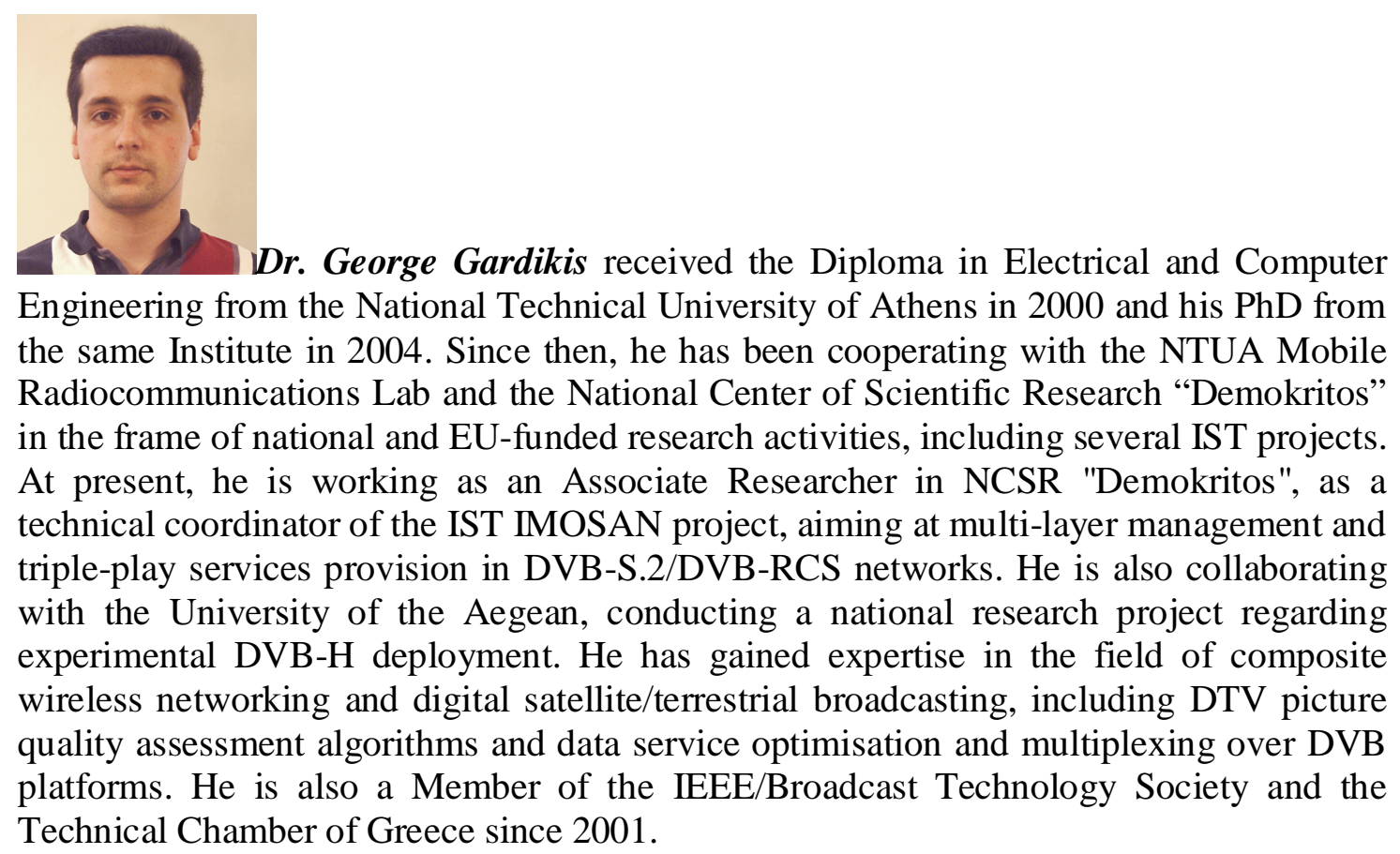


Dr. Anastasios Kourtis received his BS degree in Physics in 1978 and his Ph.D. in Telecommunications in 1984, both from the University of Athens. Since 1986 he is a researcher in the Institute of Informatics and Telecommunications of the National Centre for Scientific Research "Demokritos", currently ranking as Senior Researcher. His current research activities include, digital terrestrial interactive television, broadband wireless networks, Perceived Quality of video services, end to end QoS and real time bandwidth management in satellite communications. He is author or co-author of more than 80 scientific publications in international scientific journals, edited books and conference proceedings. Dr. Kourtis has a leading participation in many European Union funded research projects in the frame of IST/FP5/FP6 (MAMBO, SOQUET, CREDO, WIN, LIAISON, ENTHRONE). He has also coordinated three European funded Specific Targeted Research Projects (REPOSIT, ATHENA, IMOSAN).

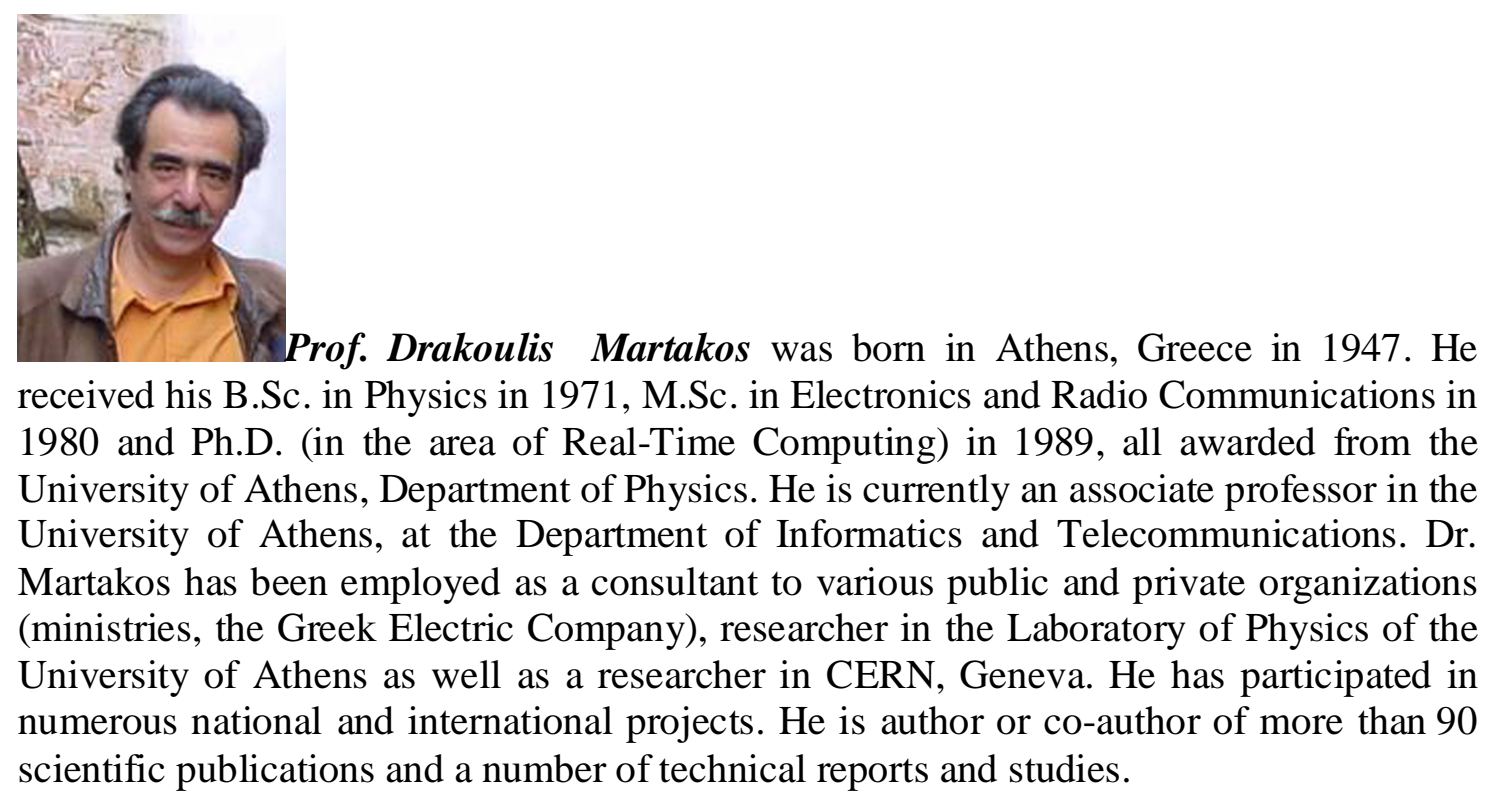

